\title{
Improved Bone Marrow Stromal Cell Adhesion on Micropatterned Titanium Surfaces*
}

\author{
Maria E. Iskandar, Aaron F. Cipriano, Jaclyn Lock, Shannon C. Gott, Masaru P. Rao and Huinan Liu
}

\begin{abstract}
Implant longevity is desired for all bone replacements and fixatives. Titanium (Ti) implants fail due to lack of juxtaposed bone formation, resulting in implant loosening. Implant surface modifications have shown to affect the interactions between the implant and bone. In clinical applications, it is crucial to improve osseointegration and implant fixation at the implant and bone interface. Moreover, bone marrow derived cells play a significant role for implant and tissue integration. Therefore, the objective of this study is to investigate how surface micropatterning on $\mathrm{Ti}$ influences its interactions with bone marrow derived cells containing mesenchymal and hematopoietic stem cells. Bone marrow derived mesenchymal stem cells (BMSC) have the capability of differentiating into osteoblasts that contribute to bone growth, and therefore implant/bone integration. Hematopoietic stem cell derivatives are precursor cells that contribute to inflammatory response. By using all three cells naturally contained within bone marrow, we mimic the physiological environment to which an implant is exposed. Primary rat bone marrow derived cells were seeded onto $T i$ with surfaces composed of arrays of grooves of equal width and spacing ranging from 0.5 to $50 \mu \mathrm{m}$, fabricated using a novel plasmabased dry etching technique. Results demonstrated enhanced total cell adhesion on smaller micrometer-scale $\mathrm{Ti}$ patterns compared with larger micrometer-scale Ti patterns, after $24-\mathrm{hr}$ culture. Further studies are needed to determine bone marrow derived cell proliferation and osteogenic differentiation potential on micropatterned $\mathbf{T i}$, and eventually nanopatterned Ti.
\end{abstract}

\section{INTRODUCTION}

Titanium (Ti), a strong, non-degradable, metallic biomaterial, has been used for orthopedic implant applications, such as joint replacement, for the past few decades. However, implant loosening caused by repeated joint loading and lack of juxtaposed bone formation often result in the Ti implant failures and revision surgeries [1]. In clinical applications, an implant is successful if it induces bone tissue integration [2]. To minimize damage induced by movement relative to the surrounding tissue and to improve overall implant efficacy, osseointegration is critically needed to stabilize orthopedic implants. It was reported that material

*Research supported by NSF BRIGE and the University of California M. E. Iskandar, A. F, Cipriano, J. Lock, and H. Liu are in the Department of Bioengineering, University of California, Riverside CA 92521 USA. S. C. Gott and M. P. Rao are in the Department of Mechanical Engineering, University of California, Riverside CA 92521 USA. (Corresponding author H. Liu, phone: 951-827-2944, fax: 951-827-6416, email: huinan.liu@ucr.edu) surface microstructure and morphology play an important role in bone cell functions and bone growth surrounding the implants [1, 3-6]. Surface properties have also shown to influence cellular adherence, migration, proliferation and differentiation [7]. Therefore, it is important to design implant surface that will support osseointegration at the implant-bone interface.

Cells derived from bone marrow play a significant role in implant and tissue integration. Therefore, the adhesion of bone marrow cells on Ti was investigated in this study.

Bone marrow stromal cells (BMSC) primarily contain mesenchymal and hematopoietic stem cells (MSC and HP, respectively). Mesenchymal and hematopoietic stem cells are precursor cells that are responsible for osteogenesis and inflammatory response, respectively. To mimic the in vivo cell response in the physiological environment, BMSCs were harvested and then seeded onto micropatterned-Ti substrates to determine how these cells respond to surface micropatterns with various sizes.

\section{MATERIALS AND METHODS}

\section{A. Fabrication of Micropatterned Ti Substrate}

A plasma dry-etching based micromachining technique was used to etch high precision patterns on each Ti substrate (Parker, 2005 \#15). Each pattern was composed of arrays of grooves of equal width with spacing ranging from 0.5 to 50 $\mu \mathrm{m}$. The patterned surface of each substrate $\left(1.7 \times 1.7 \mathrm{~cm}^{2}\right.$ total area) was divided into a nine different and orthogonally aligned subpatterns $\left(5 \times 5 \mathrm{~mm}^{2}\right)$ with different groove widths for each subpattern (Fig. 1). As a control, one of the nine subpatterns was left unpatterned (i.e., blank). Polished grade 1 Ti (99.6\% Ti, Tokyo Stainless Grinding Co., Ltd., Japan, $\mathrm{R}_{\mathrm{A}} \sim 10 \mathrm{~nm}$ RMS) wafer-based substrates were used. Before the etching process, each substrate was subjected to a standard solvent cleaning process consisting of brief ultrasonic agitation in acetone and isopropanol sequentially, followed by rinsing with deionized water and drying with $\mathrm{N}_{2}$ gas. They were then baked on a hot plate at $150{ }^{\circ} \mathrm{C}$ for 3 minutes to dehydrate the surface. A layer of photoresist was applied by spin-coating after being primed with hexamethyldisilazane, which promotes adhesion. The substrates were then lithographically patterned, followed by Cl-based dry etching and removal of the photoresist by standard solvent clean.

\section{B. Isolation of BMSC}

The BMSCs were harvested from the femur and tibia of one 17 day-old female Sprague Dawley rat weanling, after euthanasia by $\mathrm{CO}_{2}$ following an approved IACUC protocol. 


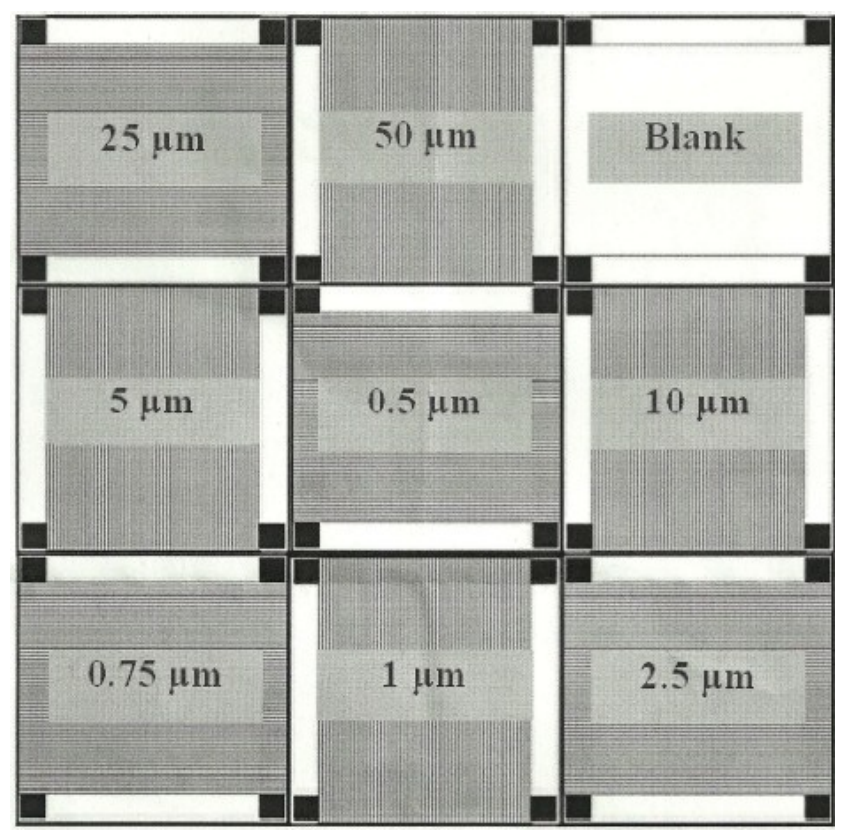

Figure 1. Schematic of paterned Ti substrate with nine different subpatterns. Labels in each subpattern denote grating feature size. The gratings in each subpattern are orthogonally oriented relative to their neighbors. The blank subpattern was not patterned and possesses surface roughness similar to that of the original substrate $\left(\mathrm{R}_{\mathrm{A}} \sim 10 \mathrm{~nm} \mathrm{RMS}\right)$.

Under standard sterile conditions, the ends of the bones were dissected and the bone marrow was flushed out of the bone cavity using Dulbecco's Modified Eagle Medium (DMEM, with high glucose) supplemented with $10 \%$ fetal bovine serum (FBS) and 1\% penicillin-streptomycin $(\mathrm{P} / \mathrm{S})$, and then collected. The collected cells in DMEM were then filtered through a $70 \mu \mathrm{m}$ nylon strainer, to remove any cell aggregates and tissue debris. The filtered cells were cultured in the supplemented DMEM under standard cell culture conditions ( $37{ }^{\circ} \mathrm{C}, 95 / 5 \%$ air/ $\mathrm{CO}_{2}$, humidified environment). The culture media was replenished as needed.

\section{Cell Seeding and Adhesion on Ti Substrates}

Before seeding, each micropatterned $\mathrm{Ti}$ substrate was ultrasonically cleaned with $70 \%$ ethanol for 10 minutes, placed in a sterile hood under ultraviolet light for $24 \mathrm{hrs}$, and then autoclaved $\left(115-120{ }^{\circ} \mathrm{C}\right)$ for $30 \mathrm{~min}$. When the bone marrow cells reached 90 to $95 \%$ confluence, they were detached from the culture substrate using trypsin and seeded onto the micropatterned-Ti substrates placed in a 6 well polystyrene tissue culture plate (PSTC) at a density of $16,000 \mathrm{cell} / \mathrm{cm}^{2}$ and incubated in DMEM supplemented with $10 \%$ FBS and $1 \% \mathrm{P} / \mathrm{S}$, under standard cell culture conditions, for $24 \mathrm{hrs}$. Cells with the supplemented DMEM and supplemented DMEM alone (without cells) were used as controls. After that time period, phosphate buffered solution (PBS) was used to remove non-adherent cells. The adherent cells were then fixed with $4 \%$ paraformaldehyde and stained with DAPI nucleic acid stain and Alexa Flour ${ }^{\circledR} 488$ cytoskeleton stain. Thereafter, the cells were imaged and the nuclei were counted using a fluorescence microscope (AMG EVOS). Nuclei were counted due to the short $24 \mathrm{hr}$ study conducted and to the difficulty in differentiating between the different cell types. Future long-term studies with specific biomarkers and larger number of samples are needed to achieve better cell analysis. The BMSC adhesion density (cells $/ \mathrm{cm}^{2}$ ) was calculated based on five or more random fields on each subpattern and control. The cell culture experiments were run in duplicate.

\section{Statistical Analysis}

The numerical data sets were analyzed using Kruskalwallis for analysis of the means (non-parametric one-way ANOVA) followed by standard post-hoc pairwise comparisons (Nemenyi test). Statistical significance was considered at $p<0.05$.

\section{RESULTS}

The Kruskal-Wallis test for non-parametric data showed significant differences between the means of cell adhesion on the patterns evaluated, the cells surrounding $\mathrm{Ti}$, and the control PSTC after the $24 \mathrm{hr}$ study $\left[\mathrm{X}^{2}(10, \mathrm{~N}=110)=\right.$ $65.3248, p<0.05]$. The post-hoc pairwise comparison test results from the rat BMSC study indicated that 25 and $50 \mu \mathrm{m}$ Ti surface pattern, and the smooth Ti surface (i.e. blank subpattern) showed significantly less adhesion compared to both the cell adhesion surrounding the $\mathrm{Ti}$ and the control PSTC (Fig. 2, 3). Furthermore, when compared with the cell adhesion density on the smooth $\mathrm{Ti}$ surface (i.e. blank subpattern), cell density was significantly greater on the 1 and $5 \mu \mathrm{m}$ Ti patterned surfaces. In addition, the $1 \mu \mathrm{m} \mathrm{Ti}$ pattern also showed significantly improved cell adhesion compared to the $25 \mu \mathrm{m} \mathrm{Ti}$ pattern. Further experiment iterations are needed on a larger number of samples to determine which of the smaller patterns show significantly superior cell adhesion.

\section{DISCUSSION}

First, it is important to consider the mechanism that leads to improved bone marrow cell adhesion on the micropatterned-Ti substrates. Studies have shown that changing the alignment and shape of cells, by controlling underlying surface features, have been related to enhanced cell functions [8]. Studies have also indicated a relationship between cell response and surface pattern that have shown to contribute to improved cell alignment and morphology. [9]. Taken together, these results emphasized the importance of the dimensions of surface patterning and suggest further research to explore optimal pattern dimensions for controlling cell functions.

In this study, bone marrow derived stromal cells were seeded and cultured on the Ti substrates in order to mimic the physiological environment to which implants are exposed, and to help provide an understanding of how different types of cells respond collectively in the body. The cell adhesion results indicated a significant reduction of cell growth on the larger patterns $(25-50 \mu \mathrm{m})$ and the smooth $\mathrm{Ti}$ surface. This in turn, points towards a preference for BMSC adhesion to the smaller range of pattern size $(0.5-10 \mathrm{um})$ on the Ti substrates. This combined with the statistical analysis results from the smaller size pattern range, which did not yield significant difference between the means of the smaller 
pattern groups and the control PSTC, support the rationale for BMSC adhesion preference for smaller patterns. Finally, the significant increase in BMSC adhesion in 1 and $5 \mu \mathrm{m}$ pattern size as compared with smooth Ti, clearly show an improved cell response that could assist with the in situ implant integration.

\section{CONCLUSION}

Results indicated a preference for BMSC adhesion on the smaller micropattern $\mathrm{Ti}$ surface but further iterations are needed to obtain statistical significance between the smaller patterns sizes. This study utilized BMSCs which contain a mixture of cells with different functions, to assess how these cells collectively interact with micropatterned Ti surfaces to simulate physiological environment in vivo. The BMSC model can serve as a clinically relevant in vitro model since it closely mimics in vivo conditions.

\section{FUTURE WORK}

Future long-term studies with specific bio-markers to differentiate between the different cell types, are needed to achieve better BMSC adhesion and proliferation analyses. In addition to cell adhesion on micropatterns, further studies on cell proliferation and differentiation are necessary to fully understand the interactions of multiple cell types with micropatterns of different size. Moreover, surface patterns in the nano-scale may facilitate differentiation of MSCs to osteoblasts [9]. Therefore, long-term cell function studies should be considered on nanopatterned $\mathrm{Ti}$ to demonstrate such behavior.

\section{ACKNOWLEDGMENTS}

The authors would like to thank the NSF BRIGE award (CBET 1125801) and the University of California for financial support. S.C.G. acknowledges the support provided by the National Science Foundation Graduate Research Fellowship Program.

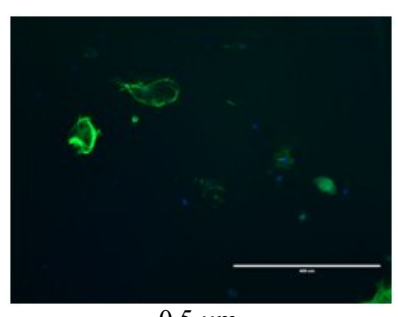

$0.5 \mu \mathrm{m}$

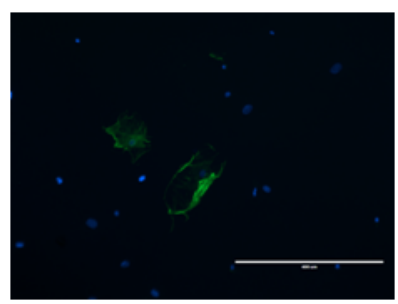

$2.5 \mu \mathrm{m}$

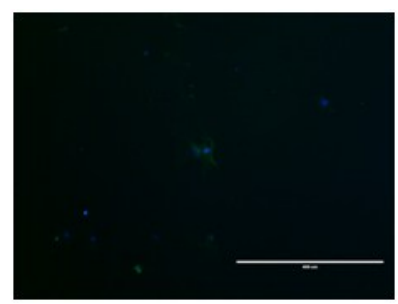

$25 \mu \mathrm{m}$

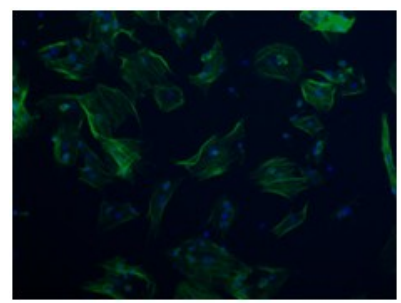

Cells Surrounding $\mathrm{Ti}$

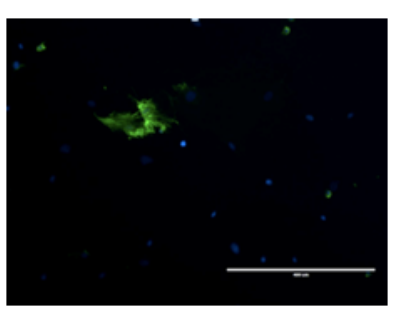

$0.75 \mu \mathrm{m}$

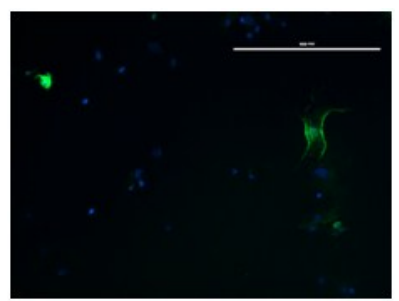

$5 \mu \mathrm{m}$

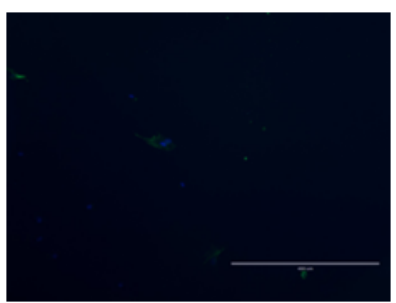

$50 \mu \mathrm{m}$

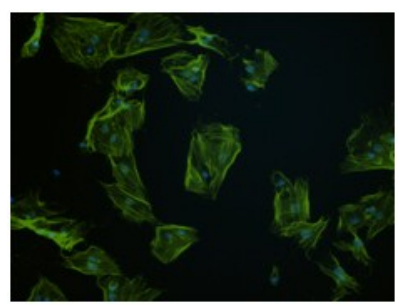

Control Cells on PSTC

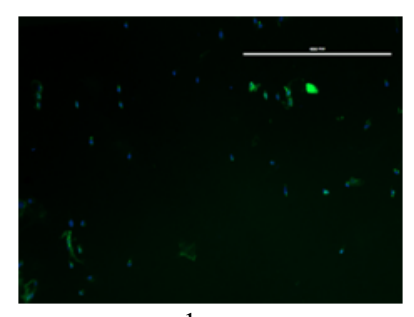

$1 \mu \mathrm{m}$

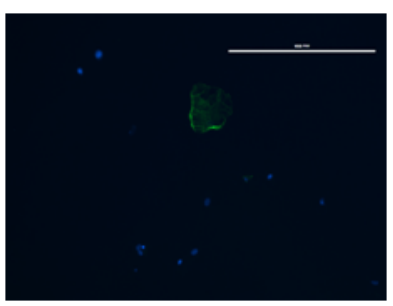

$10 \mu \mathrm{m}$

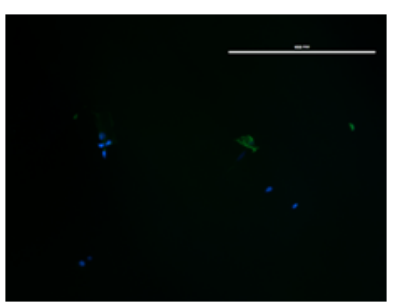

Blank

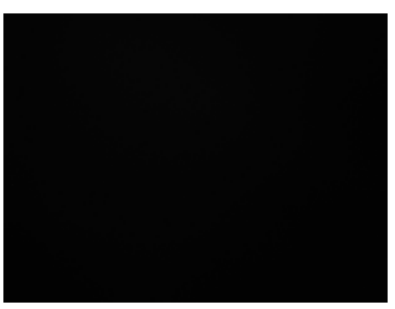

DMEM Alone (Without Cells)

Figure 2. Fluorecence images of BMSC adhesion on each micropatterned Ti surface and controls stained with DAPI and Alexa Fluor 488. Scale bar $=400$ $\mu \mathrm{m}$. 


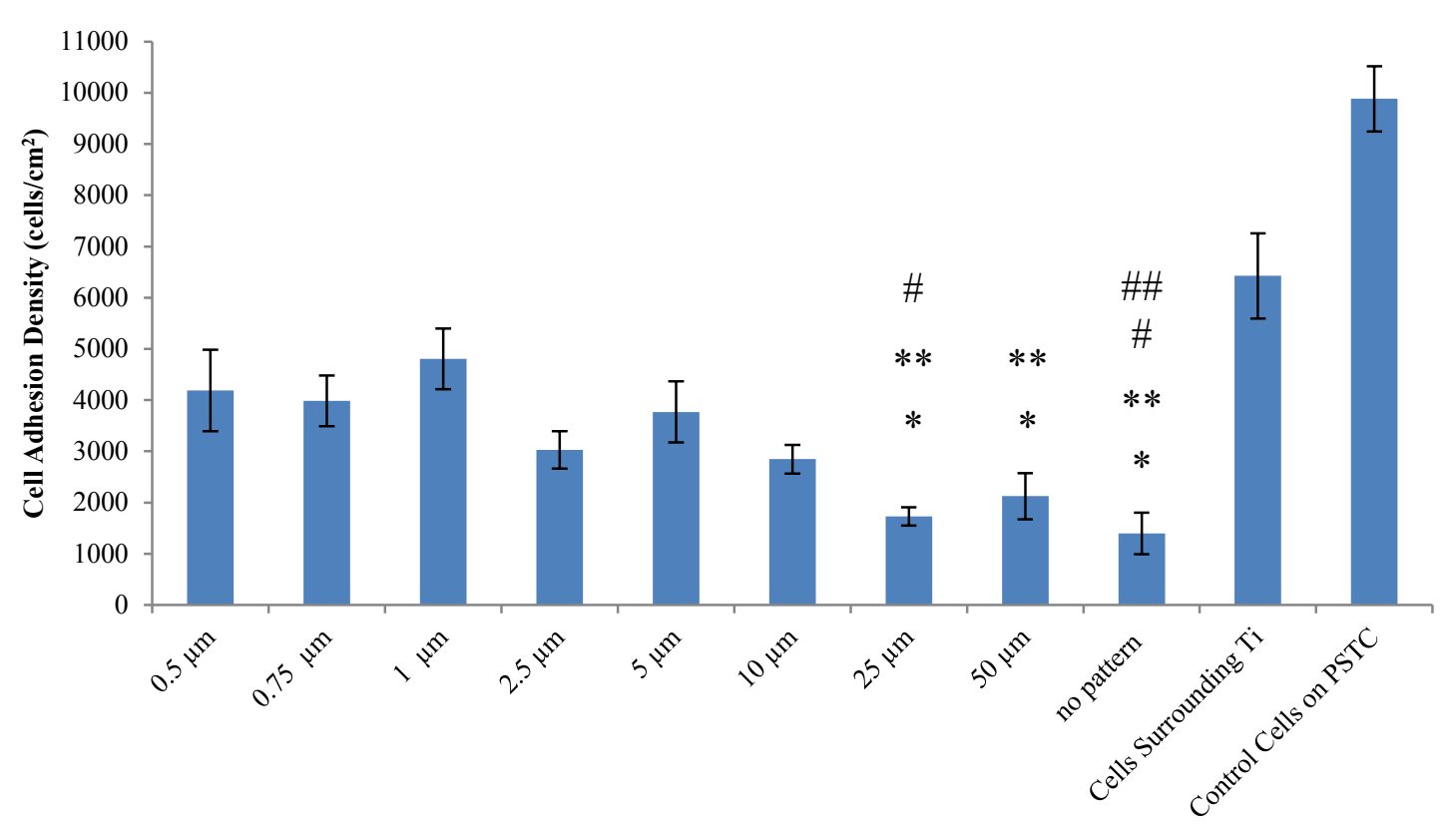

Pattern $(\mu \mathrm{m})$

Figure 3. BMSC adhesion density on the micropatterned Ti substrate of different pattern sizes and controls. ${ }^{*} \mathrm{p}<0.05$ (compared to cells surrounding Ti). ${ }^{* *} \mathrm{p}<0.05$ (compared to control cells on PSTC). ${ }^{\#} \mathrm{p}<0.05$ (compared to $1 \mu \mathrm{m}$ Ti surfaces). ${ }^{\#} \mathrm{p}<0.05$ (compared to $5 \mu \mathrm{m}$ Ti surfaces).

\section{REFERENCES}

[1] N.A. Riedel, J.D. Williams, and K.C. Popat, "Ion beam etching titanium for enhanced osteoblast response," Journal of Materials Science, vol. 46, (no. 18), pp. 6087-6095, Sep 2011.

[2] H. Liu, "The effects of surface and biomolecules on magnesium degradation and mesenchymal stem cell adhesion," Journal of Biomedical Materials Research Part A, vol. 99A, (no. 2), pp. 249-260, Nov 2011.

[3] T. Webster, C. Ergun, R. Doremus, R. Siegel, and R. Bizios, "Enhanced functions of osteoblasts on nanophase ceramics," Biomaterials, vol. 21, pp. 1803-1810, 2000.

[4] C. Ergun, H. Liu, T.J. Webster, E. Olcay, S. Yilmaz, and F.C. Sahin, "Increased osteoblast adhesion on nanoparticulate calcium phosphates with higher $\mathrm{Ca} / \mathrm{P}$ ratios," J Biomed Mater Res A, vol. 85, (no. 1), pp. 236-41, Apr 2008.

[5] C. Ergun, H.N. Liu, J.W. Halloran, and T.J. Webster, "Increased osteoblast adhesion on nanograined hydroxyapatite and tricalcium phosphate containing calcium titanate," J. Biomed. Mater. Res. Part $A$, vol. 80A, (no. 4), pp. 990-997, Mar 152007.

[6] H. Liu, E.B. Slamovich, and T.J. Webster, "Increased osteoblast functions on nanophase titania dispersed in poly-lactic-co-glycolic acid composites," Nanotechnology, vol. 16, (no. 7), pp. S601-8, Jul 2005.

[7] M. Jaeger, C. Zilkens, K. Zanger, and R. Krauspe, "Significance of nano- and microtopography for cell-surface interactions in orthopaedic implants," Journal of Biomedicine and Biotechnology, 20072007.

[8] D.E. Ingber, J.A. Madri, and J. Folkman, "Endothelial Growth-Factors and Matrix regualte DNA-Synthesis through Modulation of Cell and Nuclear Expansion," In Vitro Cellular \& Developmental Biology, vol. 23, (no. 5), pp. 387-394, May 1987.

[9] P. Clark, P. Connolly, A.S.G. Curtis, J.A.T. Dow, and C.D.W. Wilkinson, "Cell Guidance by Ultrafine Topography In Vitro," Journal of Cell Science, vol. 99, pp. 73-77, May 1991. 